\title{
OPTICAL TOMOGRAPHY IN TWO DIMENSIONS *
}

\author{
PLAMEN STEFANOV ${ }^{\dagger}$ AND GUNTHER UHLMANN ‡
}

\begin{abstract}
We consider in two dimensions, the inverse boundary problem of reconstructing the absorption and scattering coefficient of an inhomogeneous medium by probing it with diffuse light. The problem is modeled as an inverse boundary problem for the stationary linear Boltzmann equation. The information is encoded in the albedo operator. We show that we can recover the absorption and the scattering kernel from this information provided that the latter is small in an appropriate topology. We also give stability estimates and propose an approximate reconstruction procedure.
\end{abstract}

Optical Tomography concerns with the determination of spatially-varying optical absorption and scattering properties of a medium by measuring the response of the medium to transmitted near-infrared light. This has been proposed recently as a possible diagnostic tool in medicine (see $[\mathrm{A}]$ for a review). A probabilistic approach to optical tomography is the so-called diffused tomography [SGKZ]. Other areas of applications are atmospheric remote sensing [Bi], nuclear physics (see [MC] for a review), etc. We describe in more detail the mathematical problem below.

Let $X \subset \mathbf{R}^{2}$ be an open convex set with smooth boundary and consider the $2 \mathrm{D}$ stationary transport equation

$$
-v(\theta) \cdot \nabla_{x} f-\sigma(x, \theta) f+\int_{S^{1}} k\left(x, \theta^{\prime}, \theta\right) f\left(x, \theta^{\prime}\right) d \theta^{\prime}=0,
$$

where $x \in X, \theta, \theta^{\prime} \in S^{1}$ and throughout this paper we will use the notation

$$
v(\theta)=(\cos \theta, \sin \theta)
$$

Here $f(x, \theta)$ represents the density of a particle flow, $\sigma \geq 0$ is the absorption coefficient, while $k\left(x, \theta^{\prime}, \theta\right) \geq 0$ is the collision kernel. We assume that $\sigma$ and $k$ are in $L^{\infty}$. Denote $\Gamma_{ \pm}=\left\{(x, v) \in \partial X \times S^{1} ; \pm n(x) \cdot v>0\right\}$, where $n(x)$ is the outer normal to $\partial X$ at $x \in \partial X$. To ensure the solvability of the problem (1), (2), some additional conditions are needed, see i.e., [CS2]. One of them is to require that $k$ is small enough in a certain sense, for example, that $\operatorname{diam}(X)\|k\|_{L^{\infty}}<1$, see (12). In this paper we work with $k$ sufficiently small and under this assumption (1), (2) is always uniquely solvable with $f \in L^{\infty}\left(X \times S^{1}\right)$.

Let us denote by $f$ the solution to (1) satisfying the boundary condition

$$
\left.f\right|_{\Gamma_{-}}=f_{-},
$$

where $f_{-} \in C_{0}^{\infty}\left(\Gamma_{-}\right)$. The albedo operator $\mathcal{A}$ mapping the incoming flux $f_{-}$on $\Gamma_{-}$ to the outgoing one on $\Gamma_{+}$is defined as follows

$$
\mathcal{A}:\left.f_{-} \longmapsto f\right|_{\Gamma_{+}} \in L^{\infty}\left(\Gamma_{+}\right) \text {. }
$$

\footnotetext{
* Received August 13, 2002; accepted for publication April 25, 2003.

$\dagger$ Department of Mathematics, Purdue University, West Lafayette, IN 47907, USA (stefanov@math.purdue.edu). Partly supported by NSF Grant DMS-01-96440.

${ }^{\ddagger}$ Department of Mathematics, University of Washington, Seattle, WA 98195, USA (gunther@math.washington.edu). Partly supported by NSF grant DMS-00-70488 and a John Simon Guggenheim fellowship. Both authors would like to thank the hospitality of the Mathematical Sciences Research Institute where part of this work was done.
} 
The inverse problem we are interested in, is the problem of recovering $(\sigma, k)$ from knowledge of the albedo operator $\mathcal{A}$. First, if $k=0$, it is obvious that $\mathcal{A}$ does not determine uniquely $\sigma$ since in this case all we get from the knowledge of $\mathcal{A}$ is $\int \sigma(x+t(\cos \theta, \sin \theta), \theta) d t$ for any $\theta \in S^{1}$ and any $x$ and this is not enough to recover $\sigma$. For this reason in our main theorems we assume that $\sigma$ depends on $x$ only. In dimensions $n \geq 3$ this problem is formally overdetermined for the recovery of $k$ and uniqueness under the assumption above is proved in [CS1], [CS2] by studying the singularities of the distributional kernel of $\mathcal{A}$ (see also [Bo]). Some of the earlier works where this problem is treated under more restrictive assumptions are $[\mathrm{AB}],[\mathrm{CZ}],[\mathrm{L} 1]$, $[\mathrm{L} 2],[\mathrm{MC}],[\mathrm{PV}]$. Stability estimates under various assumptions are proved in [R2], [R3] (see also the references therein), [W].

In two dimensions, this inverse problem is formally determined for the recovery of $k$. Uniqueness and stability are proved for small $k$ in the case when $k=k\left(x, \cos \left(\theta-\theta^{\prime}\right)\right)$ in [R1], and in the case $k=k\left(\theta, \theta^{\prime}\right)$ uniqueness for small $k$ is proved in [T]. Note that in those cases the inverse problem is still formally overdetermined. The purpose of this paper is to prove a uniqueness results for general (small) $k\left(x, \theta^{\prime}, \theta\right)$ relative to the absorption (see Theorem 1), a stability estimate (see Theorem 2) and propose an approximate reconstruction procedure (see Remark 2).

The first main result in this paper is the following.

THEOREM 1. Define the class

$$
\mathcal{U}_{\Sigma, \varepsilon}=\left\{\left(\sigma(x), k\left(x, \theta^{\prime}, \theta\right)\right) ;\|\sigma\|_{L^{\infty}} \leq \Sigma,\|k\|_{L^{\infty}} \leq \varepsilon\right\}
$$

Then, for any $\Sigma>0$ there exists $\varepsilon>0$ such that a pair $(\sigma, k) \in \mathcal{U}_{\Sigma, \varepsilon}$ is uniquely determined by its albedo operator $\mathcal{A}$ in the class $\mathcal{U}_{\Sigma, \varepsilon}$.

REMARK 1. One can easily see from the proof (see (20) and (21)) that we can choose $\varepsilon=C(d) e^{-2 d \Sigma}$, where $C(d)>0$ and $d=\operatorname{diam}(D)$. Therefore, the smallness assumption on $k$ is relative to the size of $\sigma$. As explained above, $\varepsilon<1 / d$ guarantees that the direct problem is solvable.

The proof of Theorem 1 follows by a careful analysis of the singularities of the distribution kernel $\alpha\left(x, \theta, x^{\prime}, \theta^{\prime}\right)$ of $\mathcal{A}$ (see also [CS1], [CS2] for similar analysis in dimensions $n \geq 3$ ). In the two dimensional case we show that $\mathcal{A}$ admits the singular decomposition (see Lemma 1 and Proposition 1)

$$
\alpha=\frac{\delta\left(\theta-\theta^{\prime}\right) \delta_{\left\{x^{\prime}+\tau_{+}\left(x^{\prime}, \theta^{\prime}\right) v\left(\theta^{\prime}\right)\right\}}(x)}{n(x) \cdot v(\theta)} e^{-a\left(x^{\prime}, \theta^{\prime}\right)}+b\left(x, \theta, x^{\prime}, \theta^{\prime}\right),
$$

where $n(x)$ is the exterior normal to $\partial X$, the function $\tau_{+}\left(x^{\prime}, \theta^{\prime}\right)$ is the "exit time" defined in next section, and

$$
a\left(x^{\prime}, \theta^{\prime}\right)=\int_{0}^{\tau_{+}\left(x^{\prime}, \theta^{\prime}\right)} \sigma\left(x^{\prime}+t v\left(\theta^{\prime}\right), \theta^{\prime}\right) d t, \quad b \sin \left(\theta-\theta^{\prime}\right) \in L^{\infty} .
$$

In particular, knowing $\mathcal{A}$, we can uniquely determine $a$ and $b$. Let we have two pairs $(\sigma, k)$ and $(\tilde{\sigma}, \tilde{k})$ with albedo operators $\mathcal{A}$ and $\tilde{\mathcal{A}}$, respectively. Set

$$
\delta_{1}=\|a-\tilde{a}\|_{H^{1}\left(\Gamma_{-}\right)}, \quad \delta_{2}=\left\|(b-\tilde{b}) \sin \left(\theta-\theta^{\prime}\right)\right\|_{L^{\infty}\left(\Gamma_{-} \times \Gamma_{+}\right)} .
$$

Our second result is the following stability estimate. 
TheOrem 2. Let

$$
\mathcal{V}_{\Sigma, \varepsilon}^{s}=\left\{\left(\sigma(x), k\left(x, \theta^{\prime}, \theta\right)\right) \in H^{s}(X) \times C\left(X \times S^{1} \times S^{1}\right) ;\|\sigma\|_{H^{s}} \leq \Sigma,\|k\|_{L^{\infty}} \leq \varepsilon\right\} .
$$

Then, for any $s>1, \Sigma>0$, there exists $\varepsilon>0$ such that for any $(\sigma, k) \in \mathcal{V}_{\Sigma, \varepsilon}^{s}$ and $(\tilde{\sigma}, \tilde{k}) \in \mathcal{V}_{\Sigma, \varepsilon}^{s}$ and $0<\mu<1-1 / s$, there exists $C>0$ such that

$$
\begin{aligned}
\|\sigma-\tilde{\sigma}\|_{L^{\infty}} & \leq C \delta_{1}^{1-1 / s-\mu}, \\
\|k-\tilde{k}\|_{L^{\infty}} & \leq C\left(\delta_{1}^{1-1 / s-\mu}+\delta_{2}\right) .
\end{aligned}
$$

REMARK 2. The proofs of the theorems above are useful for reconstruction purposes as well. First, one separates the most singular term of the kernel of $\mathcal{A}$ (see (4)) from the coefficient $b$ and from the first one reconstructs $\sigma$. Next, the coefficient $b\left(x, \theta, x^{\prime}, \theta^{\prime}\right)$ is the restriction $\alpha_{1}+\alpha_{2}$ of $\phi_{1}+\phi_{2}$ for $(x, v) \in \Gamma_{+}$, see Proposition 1. We cannot separate $\alpha_{1}$ from $\alpha_{2}$ as in the case $n \geq 3$, see [CS2], but for small $k$, we have $\alpha_{2}=O\left(k^{2}\right)$, while $\alpha_{1}$ is linear in $k$ and determines directly $k$, see Proposition 1 .

1. The uniqueness, proof of Theorem 1. Recall that $\sigma$ and $k$ are $L^{\infty}$ in all variables. It is convenient to think later that $\sigma$ and $k$ are extended as 0 for $x \notin X$. Denote the left-hand side of (1) by $T f$. Denote

$$
T_{1} f=-v(\theta) \cdot \nabla_{x} f-\sigma f, \quad K f=\int_{S^{1}} k\left(x, \theta^{\prime}, \theta\right) f\left(x, \theta^{\prime}\right) d \theta^{\prime},
$$

so that $T=T_{1}+K$. First, we reduce the boundary value problem

$$
\left\{\begin{aligned}
T f & =0 \text { in } X \times S^{1} \\
\left.f\right|_{\Gamma_{-}} & =f_{-} \in C_{0}^{\infty}\left(\Gamma_{+}\right)
\end{aligned}\right.
$$

to an integral equation by a standard argument. For $(x, \theta) \in \bar{X} \times S^{1}$, define $\tau_{ \pm}(x, \theta) \geq$ 0 by the condition $\left(x \pm \tau_{ \pm}(x, \theta) v(\theta), \theta\right) \in \Gamma_{ \pm}$. Introduce the operator $M$ by

$$
M f=\int_{0}^{\infty} e^{-\int_{0}^{t} \sigma(x-s v(\theta), \theta) d s}(K f)(x-t v(\theta), \theta) d t .
$$

If we denote the solution to $T_{1} u=f,\left.u\right|_{\Gamma_{-}}=0$ by

$$
T_{1}^{-1} f=-\int_{0}^{\tau_{-}(x, \theta)} e^{-\int_{0}^{t} \sigma(x-s v(\theta), \theta) d s} f(x-t v(\theta), \theta) d t,
$$

then $M=-T_{1}^{-1} K$. Problem (8) can be written as $\left(T_{1}+K\right) f=0,\left.f\right|_{\Gamma_{-}}=f_{-}$. Applying $T_{1}^{-1}$ to the first equation, we get that $f$ solves

$$
(I-M) f=J f_{-},
$$

where

$$
J f_{-}=e^{-\int_{0}^{\tau_{-}(x, v)} \sigma(x-s v, v) d s} f_{-}\left(x-\tau_{-}(x, v) v, v\right) .
$$

Note that $J f_{-}$solves the problem $T_{1} J f_{-}=0,\left.J f_{-}\right|_{\Gamma_{-}}=f_{-}$. Then $f$ is given by

$$
f=(I-M)^{-1} J f_{-},
$$


provided that $I-M$ is invertible in a suitable space. The definition (9) of $M$ implies immediately that

$$
\|M f\|_{L^{\infty}\left(X \times S^{1}\right)} \leq C\|f\|_{L^{\infty}\left(X \times S^{1}\right)},
$$

where $C=\operatorname{diam}(X)\|k\|_{L^{\infty}}$. Therefore, if $(\sigma, k) \in \mathcal{U}_{\Sigma, \varepsilon}$ and $\varepsilon<1 / \operatorname{diam}(X)$, then $I-M$ is invertible in $L^{\infty}\left(X \times S^{1}\right)$, and then the solution $f$ to (8) is given by (11). By using Neumann series, it is not hard to see that the trace $\left.(I-M)^{-1} f\right|_{\Gamma_{+}}$is well defined in $L^{\infty}\left(\Gamma_{+}\right)$for any $f \in L^{\infty}\left(X \times S^{1}\right)$. This proves in particular that $\mathcal{A}$ maps $C_{0}^{\infty}\left(\Gamma_{-}\right)$ into $L^{\infty}\left(\Gamma_{+}\right)$under the smallness assumption on $k$ above. The same arguments also show that $\mathcal{A} f_{-}$can be defined for any $f_{-} \in L^{\infty}\left(\Gamma_{-}\right)$as well but we will not need this since we work with the distribution kernel of $\mathcal{A}$. The mapping properties of $\mathcal{A}$ in $L^{1}$ spaces are discussed in [CS2].

We proceed with the study of the distribution kernel of $\mathcal{A}$. Define first the fundamental solution $\phi\left(x, \theta, x^{\prime}, \theta^{\prime}\right)$ of the boundary value problem (8) as follows. For $\left(x^{\prime}, v^{\prime}\right) \in \Gamma_{-}$, let $\phi\left(x, v, x^{\prime}, v^{\prime}\right)$ solve

$$
\left\{\begin{aligned}
T \phi & =0 \text { in } X \times S^{1}, \\
\left.\phi\right|_{\Gamma_{-}} & =\left|n\left(x^{\prime}\right) \cdot v\left(\theta^{\prime}\right)\right|^{-1} \delta_{x^{\prime}}(x) \delta\left(\theta-\theta^{\prime}\right),
\end{aligned}\right.
$$

where $\delta_{x^{\prime}}(x) \in \mathcal{D}^{\prime}(\partial X)$ is defined by $\int_{\partial X} \delta_{x^{\prime}}(x) \varphi(x) d \mu(x)=\varphi\left(x^{\prime}\right)$, with $d \mu(x)$ the induced measure on $\partial X$. Then the albedo operator $\mathcal{A}$ has distribution kernel $\alpha\left(x, \theta, x^{\prime}, \theta^{\prime}\right)=\left.\phi\left(x, \theta, x^{\prime}, \theta^{\prime}\right)\right|_{(x, \theta) \in \Gamma_{+}},\left(x^{\prime}, \theta^{\prime}\right) \in \Gamma_{-},(x, \theta) \in \Gamma_{+}$, i.e.,

$$
\left(\mathcal{A} f_{-}\right)(x, v)=\int_{\Gamma_{-}} \alpha\left(x, \theta, x^{\prime}, \theta^{\prime}\right) f_{-}\left(x^{\prime}, \theta^{\prime}\right) d \xi\left(x^{\prime}, \theta^{\prime}\right), \quad \forall f_{-} \in C_{0}^{\infty}\left(\Gamma_{+}\right),
$$

where $d \xi\left(x^{\prime}, \theta^{\prime}\right)=\left|n\left(x^{\prime}\right) \cdot v\left(\theta^{\prime}\right)\right| d \xi\left(x^{\prime}, \theta^{\prime}\right) d \mu\left(x^{\prime}\right) d \theta^{\prime}$ and $n(x)$ is the unit exterior normal to $\partial X$.

As in [CS2], we construct a singular expansion of $\phi$ as follows. Note that for any $f_{-} \in C_{0}^{\infty}\left(\Gamma_{-}\right)$, the outgoing flux $\mathcal{A} f_{-}$is given by

$$
\mathcal{A} f_{-}=\left.J f_{-}\right|_{\Gamma_{+}}+\left.M J f_{-}\right|_{\Gamma_{+}}+\left.(I-M)^{-1} M^{2} J f_{-}\right|_{\Gamma_{+}} .
$$

Let

$$
E(x, \theta, t)=e^{\mp \int_{0}^{t} \sigma(x+s v(\theta), \theta) d s}, \quad \pm t \geq 0
$$

be the total absorption along the path $[x, x+t v(\theta)]$. Then

$$
\phi=\phi_{0}+\phi_{1}+\phi_{2},
$$

where

$$
\phi_{0}=J \phi_{-}, \quad \phi_{1}=M J \phi_{-}, \quad \phi_{2}=(I-M)^{-1} M^{2} J \phi_{-},
$$

and $\phi_{-}=\left|n\left(x^{\prime}\right) \cdot v\left(\theta^{\prime}\right)\right|^{-1} \delta_{x^{\prime}}(x) \delta\left(\theta-\theta^{\prime}\right)$ as in (13). As in [CS2],

$$
\phi_{0}=E(x, \theta,-\infty) \delta\left(\theta-\theta^{\prime}\right) \int_{0}^{\tau_{+}\left(x^{\prime}, v\left(\theta^{\prime}\right)\right)} \delta\left(x-x^{\prime}-t v\left(\theta^{\prime}\right)\right) d t .
$$

Next,

$$
\phi_{1}=\chi E\left(y, \theta^{\prime},-\infty\right) \frac{k\left(y, \theta^{\prime}, \theta\right)}{\left|\sin \left(\theta-\theta^{\prime}\right)\right|} E(y, \theta, \infty),
$$


where $y=y\left(x^{\prime}, \theta^{\prime}, x, v\right)$ is the point of intersection of the rays $(0, \infty) \ni s \mapsto x^{\prime}+s v\left(\theta^{\prime}\right)$ and $(-\infty, 0) \ni t \mapsto x+t v(\theta)$ and $\chi=\chi\left(x, \theta, x^{\prime}, \theta^{\prime}\right)$ equals 1 , if those two rays intersect in $\bar{X}$, otherwise $\chi=0$. Recall that $X$ is convex.

To estimate $\phi_{2}$ we need the following.

Lemma 1. Let $(\sigma, k)$ and $(\sigma, \tilde{k})$ be in $L^{\infty}$. Let $K, M$ and $\tilde{K}, \tilde{M}$ be related to $k$ and $\tilde{k}$ (not necessarily non-negative), respectively. Then there exists $C>0$ depending on $\operatorname{diam}(X)$ only such that

$$
\left|\left(\tilde{M} M \phi_{0}\right)\left(x, \theta, x^{\prime}, \theta^{\prime}\right)\right| \leq C\|\tilde{k}\|_{L^{\infty}}\|k\|_{L^{\infty}}\left(1+\log \frac{1}{\sin \left|\theta-\theta^{\prime}\right|}\right)
$$

almost everywhere on $X \times S^{1} \times \Gamma_{-}$, and also almost everywhere on $\Gamma_{+} \times \Gamma_{-}$.

Proof. Note first that for any $f$,

$$
|M f| \leq \int_{0}^{\infty} \int_{S^{1}}\left|k\left(x-t v(\theta), \theta^{\prime}, \theta\right) f\left(x-t v(\theta), \theta^{\prime}\right)\right| d \theta^{\prime} d t
$$

therefore

$$
\begin{aligned}
|(\tilde{K} M f)(x, \theta)| & \leq \int_{S^{1}} \int_{0}^{\infty} \int_{S^{1}}\left|\tilde{k}\left(x, \theta^{\prime \prime}, \theta\right) k\left(x-t v\left(\theta^{\prime \prime}\right), \theta^{\prime} \theta^{\prime \prime}\right) f\left(x-t v\left(\theta^{\prime \prime}\right), \theta^{\prime}\right)\right| d \theta^{\prime} d t d \theta^{\prime \prime} \\
& =\iint_{S^{1}} \frac{\left|\tilde{k}(x, \arg (x-y), \theta) k\left(y, \theta^{\prime}, \arg (x-y)\right)\right|}{|x-y|}\left|f\left(y, \theta^{\prime}\right)\right| d \theta^{\prime} d y .
\end{aligned}
$$

Here $\arg (z)$ is the polar angle of $z \in \mathbf{R}^{2}$. Setting $f=\phi_{0}$, we get

$$
\left|\left(\tilde{K} M \phi_{0}\right)\left(x, \theta, x^{\prime}, \theta^{\prime}\right)\right| \leq \int_{y \in l\left(x^{\prime}, \theta^{\prime}\right)} \frac{\left|\tilde{k}(x, \arg (x-y), \theta) k\left(y, \theta^{\prime}, \arg (x-y)\right)\right|}{|x-y|} d l(y),
$$

where $l\left(x^{\prime}, \theta^{\prime}\right)$ is the line through $x^{\prime}$ parallel to $\theta^{\prime}$ and $d l$ is the Euclidean measure on it. The following estimate

$$
\int_{-A}^{A} \frac{d s}{\sqrt{\nu^{2}+s^{2}}} \leq 2\left(1+\log \frac{A}{\nu}\right), \quad 0<\nu \leq A .
$$

is easy to prove since the integral actually equals $\log \left(A / \nu+\sqrt{1+A^{2} / \nu^{2}}\right)$. Therefore,

$$
\left|\left(\tilde{K} M \phi_{0}\right)\left(x, \theta, x^{\prime}, \theta^{\prime}\right)\right| \leq 2\|\tilde{k}\|_{L^{\infty}}\|k\|_{L^{\infty}}\left(1+\log \frac{d}{\operatorname{dist}\left\{x, l\left(x^{\prime}, \theta^{\prime}\right)\right\}}\right),
$$

where $d=\operatorname{diam}(X)$. Applying $T_{1}^{-1}$, we get

$$
\begin{aligned}
\left|\left(\tilde{M} M \phi_{0}\right)\left(x, \theta, x^{\prime}, \theta^{\prime}\right)\right| & \leq 2\|\tilde{k}\|_{L^{\infty}}\|k\|_{L^{\infty}} \int_{0}^{d}\left(1+\log \frac{d}{\operatorname{dist}\left\{x-t v(\theta), l\left(x^{\prime}, \theta^{\prime}\right)\right\}}\right) d t \\
& \leq C\|\tilde{k}\|_{L^{\infty}}\|k\|_{L^{\infty}}\left(1+\log \frac{1}{\left|\sin \left(\theta-\theta^{\prime}\right)\right|}\right),
\end{aligned}
$$

with $C=C(d)$. This proves the lemma. 
For $\phi_{2}$ we have therefore $\phi_{2}=(I-M)^{-1} \phi_{2}^{\#}$ with $\phi_{2}^{\#}=M^{2} \phi_{0}$ and by Lemma 1 ,

$$
0 \leq \phi_{2}^{\#}\left(x^{\prime}, \theta^{\prime}, x, \theta\right) \leq C\|k\|_{L^{\infty}}^{2}\left(1+\log \frac{1}{\left|\sin \left(\theta-\theta^{\prime}\right)\right|}\right) .
$$

To estimate $\phi_{2}$, write $\phi_{2}=\phi_{2}^{\#}+(I-M)^{-1} M \phi_{2}^{\#}$. Since the singularity in the r.h.s. of (17) is integrable, we get that $K \phi_{2}^{\#} \in L^{\infty}$, same for $M \phi_{2}^{\#}$, therefore $\left\|\phi_{2}-\phi_{2}^{\#}\right\|_{L^{\infty}} \leq$ $C\|k\|_{L^{\infty}}^{3}$, with some constant $C>0$, and in particular, $\phi_{2}-\phi_{2}^{\#} \in L^{\infty}$. From now on, we will denote by $C$ different positive constants. Therefore, $\phi_{2}$ also satisfies (17). Observe that $0 \leq \phi_{2}$ because $(I-M)^{-1}$ preserves the non-negative functions.

We have therefore proved the following.

Proposition 1. For $\varepsilon>0$ small enough, the fundamental solution $\phi$ of (8) defined by (13) admits the representation $\phi=\phi_{0}+\phi_{1}+\phi_{2}$ with

$$
\begin{aligned}
\phi_{0} & =E(x, \theta,-\infty) \delta\left(\theta-\theta^{\prime}\right) \int_{0}^{\tau_{+}\left(x^{\prime}, v\left(\theta^{\prime}\right)\right)} \delta\left(x-x^{\prime}-t v\left(\theta^{\prime}\right)\right) d t, \\
\phi_{1} & =\chi E\left(y, \theta^{\prime},-\infty\right) \frac{k\left(y, \theta^{\prime}, \theta\right)}{\left|\sin \left(\theta-\theta^{\prime}\right)\right|} E(y, \theta, \infty), \\
0 \leq \phi_{2} & \leq C\|k\|_{L^{\infty}}^{2}\left(1+\log \frac{1}{\left|\sin \left(\theta-\theta^{\prime}\right)\right|}\right),
\end{aligned}
$$

where $y, \chi$ are as in (15) and $C=C(\operatorname{diam}(X))$.

Fix $\Sigma>0$ and assume that we have two pairs $(\sigma, k),(\tilde{\sigma}, \tilde{k})$ in $\mathcal{U}_{\Sigma, \epsilon}$ with the same albedo operator and $\sigma, \tilde{\sigma}$ depending on $x$ only. Denote by $\phi_{j}$ and $\tilde{\phi}_{j}, j=0,1,2$, the corresponding components of the fundamental solutions $\phi$ and $\tilde{\phi}$. Then

$$
\phi_{0}+\phi_{1}+\phi_{2}=\tilde{\phi}_{0}+\tilde{\phi}_{1}+\tilde{\phi}_{2} \quad \text { for }(x, \theta) \in \Gamma_{+} .
$$

As in [CS1], the most singular terms must agree, therefore,

$$
\phi_{0}\left(x^{\prime}, \theta^{\prime}, x, \theta\right)=\tilde{\phi}_{0}\left(x^{\prime}, \theta^{\prime}, x, \theta\right) \quad \text { for }\left(x^{\prime}, \theta^{\prime}\right) \in \Gamma_{-},(x, \theta) \in \Gamma_{+} .
$$

Thus the $X$-ray transform of $\sigma(x)$ and $\tilde{\sigma}(x)$ coincide, therefore,

$$
\sigma(x)=\tilde{\sigma}(x)
$$

Next,

$$
\chi E\left(y, \theta^{\prime},-\infty\right) \frac{k\left(y, \theta^{\prime}, \theta\right)-\tilde{k}\left(y, \theta^{\prime}, \theta\right)}{\left|\sin \left(\theta-\theta^{\prime}\right)\right|} E(y, \theta, \infty)=\tilde{\phi}_{2}-\phi_{2} \quad \text { on } \Gamma_{-} \times \Gamma_{+},
$$

where $y, \chi$ are as in (15). This, together with (18), (19), leads to the inequality

$$
\begin{gathered}
\chi\left|k\left(y, \theta^{\prime}, \theta\right)-\tilde{k}\left(y, \theta^{\prime}, \theta\right)\right| \leq C\left|\sin \left(\theta-\theta^{\prime}\right)\right|\left|\tilde{\phi}_{1}-\phi_{1}\right|=C\left|\sin \left(\theta-\theta^{\prime}\right)\right|\left|\tilde{\phi}_{2}-\phi_{2}\right| \\
\text { on } \Gamma_{-} \times \Gamma_{+},
\end{gathered}
$$

where $C=e^{2 d \Sigma}, d=\operatorname{diam}(D)$. Our goal is to show that

$$
\underset{\Gamma_{-} \times \Gamma_{+}}{\operatorname{ess} \sup _{+}}\left|\sin \left(\theta-\theta^{\prime}\right)\right|\left|\tilde{\phi}_{2}-\phi_{2}\right| \leq C \varepsilon\|k-\tilde{k}\|_{L^{\infty}\left(X \times S^{1} \times S^{1}\right)}
$$


with $C>0$ depending on $\operatorname{diam}(D)$ only. Then by (20), (21),

$$
\|k-\tilde{k}\|_{L^{\infty}\left(X \times S^{1} \times S^{1}\right)} \leq C \varepsilon\|k-\tilde{k}\|_{L^{\infty}\left(X \times S^{1} \times S^{1}\right)},
$$

and for $\varepsilon>0$ small enough this implies $k=\tilde{k}$.

It remains to prove (21). By (14),

$$
\begin{aligned}
\phi_{2}-\tilde{\phi}_{2} & =(I-M)^{-1} \phi_{2}^{\#}-(I-\tilde{M})^{-1} \tilde{\phi}_{2}^{\#} \\
& =(I-M)^{-1}\left(M^{2} \phi_{0}-\tilde{M}^{2} \phi_{0}\right)+(I-\tilde{M})^{-1}(M-\tilde{M})(I-M)^{-1} \tilde{\phi}_{2}^{\#} \\
& =I_{1}+I_{2} .
\end{aligned}
$$

Writing $M^{2}-\tilde{M}^{2}=M(M-\tilde{M})+(M-\tilde{M}) \tilde{M}$, the first term $I_{1}$ can be estimated by Lemma 1 and the paragraph after (17), thus

$$
\left|I_{1}\right| \leq C \varepsilon\|k-\tilde{k}\|_{L^{\infty}}\left(1+\log \frac{1}{\left|\sin \left(\theta-\theta^{\prime}\right)\right|}\right) .
$$

To estimate $I_{2}$, write

$$
\left|I_{2}\right| \leq C\|k-\tilde{k}\|_{L^{\infty}} \int_{S^{1}}\left\|(I-M)^{-1} \tilde{\phi}_{2}\right\|_{L^{\infty}(X)} d \theta \leq C \varepsilon^{2}\|k-\tilde{k}\|_{L^{\infty}} .
$$

By (22), (23), (24),

$$
\left|\phi_{2}-\tilde{\phi}_{2}\right| \leq C \varepsilon\|k-\tilde{k}\|_{L^{\infty}}\left(1+\log \frac{1}{\left|\sin \left(\theta-\theta^{\prime}\right)\right|}\right) .
$$

for a.e. $\left(x, \theta, x^{\prime}, \theta^{\prime}\right) \in X \times S^{1} \times \Gamma_{-}$and for a.e. $\left(x, \theta, x^{\prime}, \theta^{\prime}\right) \in \Gamma_{+} \times \Gamma_{-}$. The last statement follows from the fact that $M=T_{1}^{-1} K$ and from the smoothing properties of $T_{1}^{-1}$. Since $\sin \left|\theta-\theta^{\prime}\right| \log \left(1 / \sin \left|\theta-\theta^{\prime}\right|\right)$ is bounded, this proves (21).

This completes the proof of Theorem 1 .

2. The stability estimate, proof of Theorem 2. According to Proposition 1 , for the distribution kernel $\alpha\left(x, \theta, x^{\prime}, \theta^{\prime}\right)$ of $\mathcal{A}$ we have the representation (4), where $a$ is as in (5), while $b=\left.\left(\phi_{1}+\phi_{2}\right)\right|_{(x, \theta) \in \Gamma_{+}}=: \alpha_{1}+\alpha_{2}$. Then $b$ is a function and an elementary calculation show that $b \in L^{\infty}\left(\Gamma_{+}, L^{1}\left(\Gamma_{-}\right)\right)$. Proposition 1 shows that $b \sin \left(\theta-\theta^{\prime}\right) \in L^{\infty}$. Assume that we have two pairs of continuous functions $(\sigma, k)$ and $(\tilde{\sigma}, \tilde{k})$ in $\mathcal{V}_{\Sigma, \varepsilon}^{s}$ with albedo operators $\mathcal{A}$ and $\tilde{\mathcal{A}}$, respectively. In what follows we will denote the quantities $a, b$, $\alpha$, etc., related to the second pair by putting a tilde sign over it. Also, we will use the notation $\Delta \mathcal{A}$ to denote the difference $\Delta \mathcal{A}=\mathcal{A}-\tilde{\mathcal{A}}$, and similarly $\Delta a=a-\tilde{a}$, etc. In this paper, $\Delta$ never stands for the Laplacian.

Let $\delta_{1}$ and $\delta_{2}$ be as in (6). According to Proposition $1, \delta_{2}<\infty$. Our goal is to estimate $\Delta \sigma$ and $\Delta k$ in terms of $\delta_{1}$ and $\delta_{2}$. Observe that, as in the uniqueness proof, $\Delta e^{-a}$ and $\Delta\left(\alpha_{1}+\alpha_{2}\right)$ can be recovered from $\Delta \alpha$ by separating the most singular part of $\Delta \alpha$ from the rest. Therefore, $\delta_{1}$ measures the magnitude of the singular part of $\Delta \alpha$, while $\delta_{2}$ measures the magnitude of the regular part.

We start with estimating $\Delta \sigma$. By a result of Mukhometov [M],

$$
\|\Delta \sigma\|_{L^{2}} \leq C \delta_{1} .
$$

Next we estimate $\Delta k$ in terms of $\delta_{1}$ and $\delta_{2}$. Set $E_{1}\left(y, \theta, \theta^{\prime}\right)=E\left(y, \theta^{\prime},-\infty\right) E(y, \theta, \infty)$. Then by (15), $\sin \left|\theta-\theta^{\prime}\right| \alpha_{1}\left(x, \theta, x^{\prime}, \theta^{\prime}\right)=\chi\left(E_{1} k\right)\left(y, \theta^{\prime}, \theta\right)$ with $y, \chi$ as in (15). Our 
starting point is the relation $\Delta\left(E_{1} k\right)=k \Delta E_{1}+\tilde{E}_{1} \Delta k$. By the inequality $\mid e^{-s_{1}}-$ $e^{-s_{2}}|\leq| s_{1}-s_{2} \mid$ for $0 \leq s_{i}, i=1,2$, we get that $\left|\Delta E_{1}\right| \leq 2 d|\Delta \sigma| \leq 2 d \delta_{1}^{\prime}$, where

$$
\delta_{1}^{\prime}=\|\Delta \sigma\|_{L^{\infty}} .
$$

Hence, on $\operatorname{supp} \chi$,

$$
\begin{aligned}
\tilde{E}_{1}|\Delta k|\left(y, \theta, \theta^{\prime}\right) & \leq\left|\Delta\left(E_{1} k\right)\right|+k\left|\Delta E_{1}\right| \\
& \leq\left|\Delta\left(\alpha_{1}+\alpha_{2}\right) \sin \right| \theta-\theta^{\prime}\left|-\Delta \alpha_{2} \sin \right| \theta-\theta^{\prime}||+C \delta_{1}^{\prime} \\
& \leq \delta_{2}+\left|\Delta \alpha_{2}\right| \sin \left|\theta-\theta^{\prime}\right|+C \delta_{1}^{\prime} .
\end{aligned}
$$

Therefore,

$$
\|\Delta k\|_{L^{\infty}} \leq C\left(\delta_{1}^{\prime}+\delta_{2}+\left\|\Delta \alpha_{2} \sin \left|\theta-\theta^{\prime}\right|\right\|_{L^{\infty}}\right)
$$

We want to prove an estimate similar to (21) on the last term in the r.h.s. above. Recall that in (21), we have $\sigma=\tilde{\sigma}$, while here we only know how to estimate the difference $\Delta \sigma=\sigma-\tilde{\sigma}$. Nevertheless, we can proceed along similar lines. Denote by $\gamma$ the trace on $\Gamma_{+}$. Clearly, $\gamma: C\left(\bar{X} \times S^{1}\right) \rightarrow C\left(\Gamma_{+}\right)$is bounded with norm 1. Note also that since we assume here that $\sigma$ and $k$ are continuous, $(I-M)^{-1}$ exists in $C\left(X \times S^{1}\right)$ for $\varepsilon \ll 1$. Similarly to $(21)$,

$$
\begin{aligned}
\Delta \alpha_{2} & =\gamma(I-M)^{-1} \phi_{2}^{\#}-\gamma(I-\tilde{M})^{-1} \tilde{\phi}_{2}^{\#} \\
& =\gamma(I-M)^{-1}\left(M^{2} \phi_{0}-\tilde{M}^{2} \tilde{\phi}_{0}\right)+\gamma(I-\tilde{M})^{-1}(M-\tilde{M})(I-M)^{-1} \tilde{\phi}_{2}^{\#} \\
& =I_{1}+I_{2} .
\end{aligned}
$$

To estimate $I_{1}$, we write $M^{2} \phi_{0}-\tilde{M}^{2} \tilde{\phi}_{0}=\left(M^{2}-\tilde{M}^{2}\right) \phi_{0}+\tilde{M}^{2} \Delta \phi_{0}$. In view of (22), (23), the contribution to $I_{1}$ of the first term in the r.h.s above can be estimated similarly to (23) with $\|\Delta k\|_{L^{\infty}}$ there replaced by $\|\Delta k\|_{L^{\infty}}+\|\Delta \sigma\|_{L^{\infty}}$. Next, to estimate the contribution of $\tilde{M}^{2} \Delta \phi_{0}$ to $I_{1}$, notice first that $\Delta \phi_{0}$ is given by the first formula in Proposition 1 , with the factor $E(x, \theta,-\infty)$ there replaced by $\Delta E(x, \theta,-\infty)$ and the latter is $O\left(\delta_{1}^{\prime}\right)$. As in Lemma 1 , this easily implies that $\left|\tilde{M}^{2} \Delta \phi_{0}\right|=O\left(\delta_{1}^{\prime}\right) /(1-$ $\left.\log \sin \left|\theta-\theta^{\prime}\right|\right)$, hence $\left|\gamma(I-M)^{-1} \tilde{M}^{2} \Delta \phi_{0}\right|=O\left(\delta_{1}^{\prime}\right) /\left(1-\log \sin \left|\theta-\theta^{\prime}\right|\right)$. This shows that we have the following analogue of (23)

$$
\left|I_{1}\right| \leq C \varepsilon\left(\|\Delta k\|_{L^{\infty}}+\delta_{1}^{\prime}\right)\left(1+\log \frac{1}{\left|\sin \left(\theta-\theta^{\prime}\right)\right|}\right) .
$$

In a similar way, we obtain the version of (24) in this case

$$
\left|I_{2}\right| \leq C \varepsilon^{2}\left(\|\Delta k\|_{L^{\infty}}+\delta_{1}^{\prime}\right),
$$

Combining (28), (29) and (30), we get, similarly to (21),

$$
\left|\Delta \alpha_{2} \sin \right| \theta-\theta^{\prime} \mid \|_{L^{\infty}} \leq C \varepsilon\left(\|\Delta k\|_{L^{\infty}}+\delta_{1}^{\prime}\right)
$$

Therefore, for $\varepsilon>0$ small enough, (27) implies the estimate

$$
\|\Delta k\|_{L^{\infty}} \leq C\left(\delta_{1}^{\prime}+\delta_{2}\right) .
$$

Estimate (32) is the base of our stability estimate. We are going now to use the interpolation inequality

$$
\|f\|_{\beta_{1} s_{1}+\beta_{2} s_{2}} \leq\|f\|_{s_{1}}^{\beta_{1}}\|f\|_{s_{2}}^{\beta_{2}}, \quad \beta_{1}+\beta_{2}=1, \beta_{j} \geq 0,
$$


where, in our case, $\|\cdot\|_{s}$ will be the norm in the Sobolev space $H^{s}(X)$. Let $\sigma$ and $\tilde{\sigma}$ belong to $\mathcal{V}_{\Sigma, \varepsilon}^{s}$. Then

$$
\|\Delta \sigma\|_{1+s \mu} \leq C(\Sigma)\|\Delta \sigma\|_{L^{2}}^{1-1 / s-\mu}
$$

for any fixed $0 \leq \mu \leq 1-1 / s$. By a standard Sobolev embedding theorem and (25),

$$
\delta_{1}^{\prime}=\|\Delta \sigma\|_{L^{\infty}} \leq C(\Sigma)\|\Delta \sigma\|_{L^{2}}^{1-1 / s-\mu} \leq C^{\prime}(\Sigma) \delta_{1}^{1-1 / s-\mu} .
$$

Therefore, (32) yields

$$
\|\Delta k\|_{L^{\infty}} \leq C\left(\delta_{1}^{1-1 / s-\mu}+\delta_{2}\right)
$$

Estimates (33) and (34) complete the proof of Theorem 2.

\section{REFERENCES}

[A] S. Arridge, Optical tomography in medical imaging, Inverse problems, 15 (1999), R41R93.

[AB] Yu. E. Anikonov, B. A. Bubnov, Inverse problems of transport theory, Soviet Math. Doklady, 37 (1988), pp. 497-499.

[Bi] S. Bissonette, Imaging through fog and rain, Opt. Eng., 31 (1992), pp. 1045-1052.

[Bo] A. BondarEnKo, The structure of the fundamental solution of the time-independent transport equation, J. Math. Anal. Appl., 221:2 (1998), pp. 430-451.

[CS1] M. Choulli AND P. Stefanov, Reconstruction of the coefficients of the stationary transport equation from boundary measurements, Inverse Prob., 12 (1996), L19-L23.

[CS2] M. Choulli And P. Stefanov, An inverse boundary value problem for the stationary transport equation, Osaka J. Math., 36:1 (1999), pp. 87-104.

[CZ] M. Choulli, A. Zeghal, Laplace transform approach for an inverse problem, Transport Theory and Stat. Phys., 24:9 (1995), pp. 1353-1367.

[L1] E. W. LARSEn, Solution of multidimensional inverse transport problems, J. Math. Phys., 25:1 (1984), pp. 131-135.

[L2] E. LARSEN, Solution of three-dimensional inverse transport problems, Transport Theory Statist. Phys., 17 (1988), No. 2-3, pp. 147-167.

[MC] N. J. MCCormick, Recent developments in inverse scattering transport methods, Trans. Theory and Stat. Phys., 13 (1984), pp. 15-28.

[M] R. Muknometov, On the problem of integral geometry, Math. Problems of Geophysics, Akad. Nauk SSSR, Sibirs. Otdel., Vychisl. Tsentr, Novosibirsk, 6:2 (1975), pp. 212-242 (in Russian).

[PV] A. I. PRILEPKO, N. P. Volkov, Inverse problems for determining the parameters of nonstationary kinetic transport equation from additional information on the traces of the unknown function, Differentsialnye Uravneniya, 24 (1988), pp. 136-146.

[R1] V.G. Romanov, Estimation of stability in the problem of determining the attenuation coefficient and the scattering indicatrix for the transport equation, Sibirsk. Mat. Zh., 37:2 (1996), pp. 361-377, iii; translation in Siberian Math. J., 37:2 (1996), pp. 308-324.

[R2] V.G. Romanov, Stability estimates in the three-dimensional inverse problem for the transport equation, J. Inverse Ill-Posed Probl, 5:5 (1997), pp. 463-475.

[R3] V. Romanov, A stability theorem in the problem of the joint determination of the attenuation coefficient and the scattering indicatrix for the stationary transport equation, Mat. Tr., 1:1 (1998), pp. 78-115.

[SGKZ] J. R. Singer, F. A. Grünbaum, P. Kohn, And J. P. Zubelli, Image reconstruction of the interior of bodies that diffuse radiation, Science, 248 (1990), pp. 990-993.

[T] A. TAMAsan, An inverse boundary value problem in two dimensional transport, Inverse Problems, 18 (2002), pp. 209-219.

[W] J. WANG, Stability estimates of an inverse problem for the stationary transport equation, Ann. Inst. H. Poincaré Phys. Théor., 70:5 (1999), pp. 473-495. 
P. STEFANOV AND G. UHLMANN 